\title{
Anatomic reconstruction of the medial collateral ligament in multi-ligaments knee injury using achilles allograft : a modification of Marx's technique
}

\author{
Hamidreza Yazd, John Y. Kwon, Mohammad Ghorbanhoseini, Alireza Yousof Gomrokchi, Paniz Motaghi
}

From the Firoozgar Hospital, Neuromusculoskeletal research center, Iran University of Medical Sciences, Tehran, Iran

\begin{abstract}
Medial Collateral Ligament (MCL) injury may require operative treatment. Marx et al. described the latest technique for reconstruction of MCL. While good results have been reported using the Marx technique, some issues have been observed. To address the mentioned issues, a modification to the Marx technique has been devised.

Eleven patients were enrolled and their ligaments were repaired by the fixation of allograft on the proximal and distal attachment footprints of the superficial MCL. For preventing loss of knee ROM, MCL and other ligaments were reconstructed in 2 separate stages. At the last follow up the ROM, knee ligament laxity and functional outcome scores, subjective (IKDC) and Lysholm score were evaluated and recorded.

Knee motion was maintained in all cases. Two cases demonstrated 1+ valgus instability at 30 degrees of knee flexion. Both were treated for combined MCL and PCL tear, the rest were stable. The average IKDC-subjective score was $93 \pm 4$ and the average Lysholm score was $92 \pm 3$. All patients were satisfied and returned to their previous level of activity.

In this technique, the superficial MCL was reconstructed closer to its anatomical construct. Patients didn't have any complaints of hardware under the skin and the need for a second surgery for hardware removal was avoided. Patients didn't have any complaints of hardware under the skin and the need for a second surgery for hardware removal was avoided. Also reconstructing the ligaments in 2 stages helped to preserve the knee motion.

Level of Evidence : Level IV therapeutic
\end{abstract}

No benefits or funds were received in support of this study. None of the authors have a conflict of interest.
Keywords : MCL repair ; allograft ; Marx's technique ; superficial MCL ; anchor sutures ; staged reconstruction.

\section{INTRODUCTION}

The medial collateral ligament (MCL) of the knee is the primary stabilizer that resists valgus deforming forces. It provides $80 \%$ of the valgus stability in 30 degrees of knee flexion and $60 \%$ at full extension. Other elements that play a role in valgus stability at full extension are the anterior

Hamidreza Yazdi ${ }^{1}$, MD, Associated Professor,

John Y. Kwon², MD, Assistant Professor,

- Mohammad Ghorbanhoseini, MD, Research Scholar, Orthopedic Surgeon,

- Alireza Yousof Gomrokchi ${ }^{3}, \mathrm{MD}$,

- Paniz Motaghi ${ }^{3}, \mathrm{MD}$.

${ }^{1}$ Department of knee surgery, Firoozgar Hospital, Neuromusculoskeletal research center, Iran University of Medical Sciences, Tehran, Iran.

${ }^{2}$ Harvard Medical School, BIDMC, Carl J. Shapiro Department of Orthopaedics, 330 Brookline Avenue Boston, MA 02215 .

${ }^{3}$ Shafa Yahyaeian Hospital, Iran University of Medical Sciences, Tehran, Iran.

Correspondence : Alireza Yousof Gomrokchi, Shafa Yahyaeian Hospital, Iran University of Medical Sciences, Tehran, Iran, District 12, Mojahedin Eslam St, Tehran, Iran. Phone : +98 912 7826840, Fax: +98 2133542001.

Email : arugomrokchi@gmail.com

- 2021, Acta Orthopædica Belgica. 
cruciate ligament (ACL), the posteromedial capsule and the posterior oblique ligament $(13,22,24,30)$. Additionally, structures that are considered static stabilizers of the medial knee include the superficial and deep MCL as well as the posterior oblique ligament (24).

Although most patients who sustain MCL injuries regain their activity level with non-operative treatment (24), severe cases with multiple ligament injuries and those with isolated symptomatic MCL laxity may require operative treatment $(24,16,1,29)$.

Treatment of MCL associated with ACL injury, one of the most frequently combined ligamentous injuries of the knee, has been associated with a relatively high incidence of postoperative arthrofibrosis. Therefore, it has been suggested by some authors to reconstruct combined ACL and MCL injury in two stages to avoid further complications (4).

Several techniques for MCL reconstruction have been described (23). Some surgeons use semitendinosus autograft with preservation of the tibial insertion $(22,1,5,19)$, while others prefer using allograft $(4,10)$. Double-bundle reconstruction, compared to a single-bundle reconstruction, is another oft utilized technique, although it can be technically demanding. There are multiple attachment sites on the femur as well as on the tibia, more graft tissue is needed, and multiple fixation devices like staples, screws, and washers are required $(22,23,4,15,29)$.

Marx et al. have described the latest technique for reconstruction of the MCL (22). They used Achilles tendon allograft and reconstructed the MCL at the same time as the ACL and secured the allograft by fixing the bone block attached to the allograft into the tibia using a metallic screw and washer (22).

Although Marx's technique was effective in MCL reconstruction, there are some considerations and issues to contend with. For instance, after securing the distal part of the graft $5-7 \mathrm{~cm}$ below the joint line on the medial tibia, the graft may not lie in contact with the underlying bone and may remain tented over the bone. Additionally, some patients complain of hardware prominence. Furthermore, Marx reported a loss of range of motion in several patients, especially those who had multiple ligament injuries. Marx mentioned finding the location for distal attachment of the allograft by checking isometry. However, this is rather subjective and possibly imprecise.

To resolve these issues, we have developed a modification of Marx's technique. This study aims to describe a new approach and evaluate the results of MCL reconstruction with the modified Marx technique.

\section{PATIENTS \& METHODS}

Local institutional review board approval was obtained for the conduction of this study. From February 2013 to January 2015, eleven patients who had suffered MCL tears in addition to ACL, PCL or both ACL and PCL tears and were candidates for surgical reconstruction were recruited for this study. ( 1 female and 10 males)

The mean age was 32 years old (range 26-38 years old). Mean follow up was 19 months (range 12-27 months).

All cases enrolled had multi-ligaments injury of the knee. Six patients with MCL+ACL, two with MCL+ PCL and three with MCL+ACL + PCL tears.

The criteria for MCL reconstruction was residual medial knee instability after failure of conservative treatment in patients with ACL and MCL injuries, and all patients with PCL or PCL and ACL injuries with MCL instability of $3+$ or more. Infection, severe soft tissue trauma at the time of proposed surgery, and significant medical comorbidities were contraindications for surgery.

During the first 7 to 10 days after the trauma, when the soft tissue and patient's general condition was improved, the first stage of MCL reconstruction was performed. Patients were encouraged to use a functional knee brace and start a progressive knee range of motion within 2 weeks. They were also allowed to partial weight bear as tolerated with 2 crutches in 4-6 weeks after surgery and then full weight bear with a knee brace. Muscles strengthening and proprioceptive exercises were advised.

Approximately 8 to 10 weeks after the first stage, when the patient regained full knee ROM and showed acceptable neuromuscular control, the second stage for anatomic reconstruction of all other 


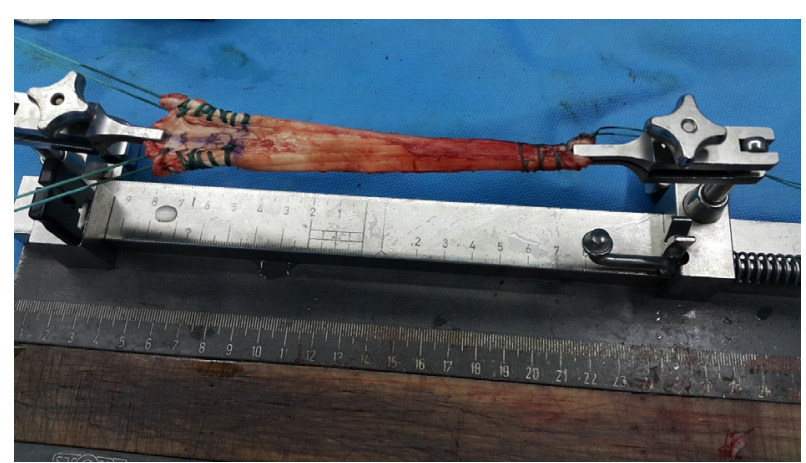

Figure 1. - Preparation of the allograft by applying appropriate tension and placement of nonabsorbable sutures.

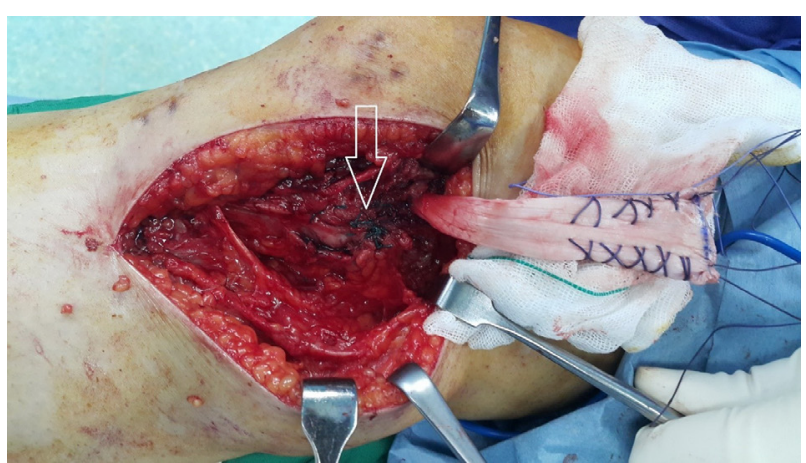

Figure 2. - The allograft is fixed into the femoral socket with an absorbable interference screw. The posteromedial capsule is reefed (white arrow).

ligaments were done. The rehabilitation program under the supervision of a physiotherapist was done early after surgery.

Patients were appropriately followed during their postoperative course. The following information was recorded at the final follow up: follow up duration, range of motion, side-to-side ligament laxity differences and functional outcome scores. ACL laxity was assessed by the Lachman, anterior drawer and pivot shift tests. The PCL was assessed with the posterior drawer test. MCL laxity was assessed with a valgus stress test at 0 and 30 degrees of knee flexion. All assessments were compared to the contralateral side and differences were recorded. Functional outcome scores utilized included the International Knee Documentation Committee (IKDC) subjective knee score and Lysholm knee score.

The surgical technique used in this research is based on Marx's Technique with some modifications.
The patient was positioned supine on a universal surgical bed. All surgeries were performed under general or spinal anesthesia. Achilles tendon allograft with a length of $12-14 \mathrm{~cm}$ was utilized. The $20 \mathrm{~mm}$ distal insertional Achilles tendon was trimmed to a width of $8 \mathrm{~mm}$. The broad proximal portion of the allograft tendon was then sutured on both sides using a non-absorbable suture (Fig. 1). A long medial longitudinal skin incision was made and skin, subcutaneous fascia and the first layer of the medial side of the knee developed. The anatomical footprints of the MCL on the femoral and tibial sides were located, and then a guide pin was inserted 3 to $5 \mathrm{~mm}$ proximal and posterior to the medial femoral epicondyle and just at the center of the femoral footprint. This pin was then guided parallel to the joint line under fluoroscopic guidance and in a 10-15 degree anterior direction to avoid the intercondylar notch. The femoral tunnel was made by reaming over the guide pin with an 8-mm diameter reamer up to a depth of $20 \mathrm{~mm}$, and the prepared Achilles allograft was inserted into the femoral socket and fixed with an absorbable interference screw (Arthrex Bio-Interference screw, Naples, FL) (Fig. 2). Two $5 \mathrm{~mm}$ anchor sutures (Depuy, Warsaw, IN) were placed $1.5-2 \mathrm{~cm}$ apart on the tibial footprint of superficial MCL approximately $5-7 \mathrm{~cm}$ below the joint line. If localization of the anatomic footprint was difficult, screws were inserted posterior and inferior to the pes anserinus on the posterior half of tibia. Another anchor suture was then inserted into the posterior half of the tibia $10 \mathrm{~mm}$ below

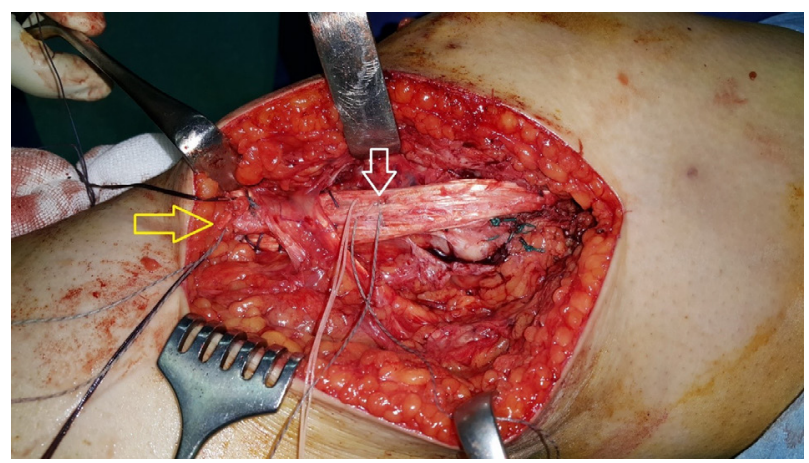

Figure 3. - Anchor sutures are placed at proximal superficial MCL attachment (white arrow) and distal superficial MCL attachment (yellow arrow), and sutures are passed through the allograft. 


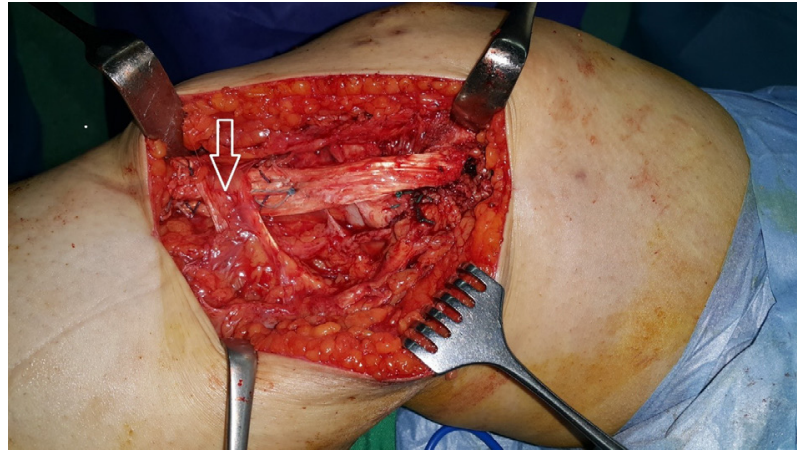

Figure 4. - Allograft is fixed and sutures are tied. Note that the allograft is passed under the pes anserinus tendons (white arrow).

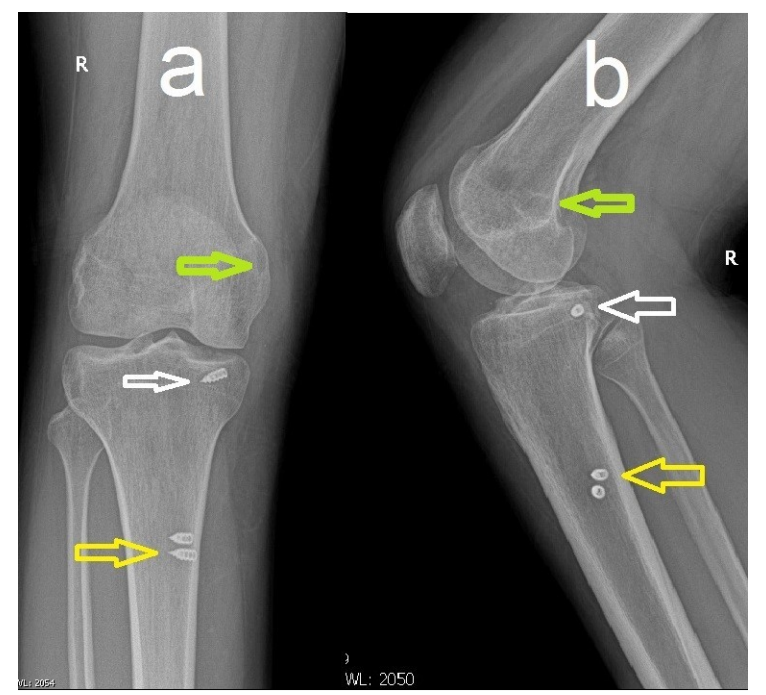

Figure 5. - Anterior-Posterior (a) and lateral (b) X-rays confirm tunnel position and hardware placement. One anchor suture is placed at the proximal attachment of superficial MCL (white arrow) and 2 suture anchors at the distal attachment of superficial MCL (yellow arrow). Note the position of the femoral tunnel (green arrow).

the joint line at the proximal attachment site of the superficial MCL. The graft was passed under the pes anserinus tendons and over the anatomic footprint and fixed using two anchor sutures with optimal tension at the 20-30 degrees of knee flexion under varus force (Fig. 3). Finally, the proximal suture was fixed over the graft (Fig. 4). In seven cases that showed posteromedial laxity, the capsule was reefed by using non-absorbable sutures before tibial graft fixation. Subcutaneous tissue and skin closure were performed in standard fashion and tunnel position and hardware placement were confirmed with postoperative radiographs (Fig. 5).

Ethics approval and consent to participate : The study was approved by the institutional review board (IRB) of Firoozgar Hospital. Informed consent were obtained from each patient before admission.

\section{RESULTS}

At final follow up, no patients were found to have gross malalignment or gait abnormalities as assessed clinically and no complications were reported. All patients reported being satisfied and reported excellent results. The ROM was full in all cases. No complaints of hardware irritation were noted.

Side-to-side MCL integrity showed a firm endpoint on valgus stress test with no gross sideto-side differences at full extension in all the cases. Two cases demonstrated $1+$ valgus instability at $30^{\circ}$ of knee flexion. Both were treated for combined MCL and PCL tear.

Average IKDC-subjective scores demonstrated statistically significant improvements from $63 \pm 2$ to $93 \pm 4$ (P value $<0.05)$. Lysholm scores demonstrated statistically significant improvements from $67 \pm 4$ to $92 \pm 3(\mathrm{P}$ value $<0.05)$ after surgery.

\section{DISCUSSION}

The superficial MCL is the largest structure of the medial part of the knee and originates from 3.2 $\mathrm{mm}$ proximal and $4.8 \mathrm{~mm}$ posterior to the medial epicondyle at the center of knee motion on the medial femoral epicondyle of the femur $(22,24,8,26)$. According to La Prade, et al. and Brantigan, et al., the superficial MCL has 2 attachments on the tibia $(17,6,7)$. The proximal attachment of the superficial MCL inserts directly over the anterior arm of the semimembranosus approximately $1 \mathrm{~cm}$ below the knee $(17,6,7)$, and the distal attachment inserts on the proximal tibia just anterior to the posteromedial crest of the tibia and posterior to the pes anserine insertion, five to seven centimeters $(\mathrm{cm})$ below the joint line, with an average length of $11 \mathrm{~cm}(10-12$ $\mathrm{cm})$ and an average width of $1.5 \mathrm{~cm}(22,24,8,7,28)$. The 
superficial MCL consists of anterior and posterior portions that play different rolls anatomically $(24,4)$. The deep MCL is the thick part of the middle third of the medial capsule, also known as the middle capsular ligament which originates inferior to the medial epicondyle of the femur and inserts on the tibia $1 \mathrm{~cm}$ below the joint line (22,24). MCL also provides resistance to external rotational forces to the lower extremity (4).

Ligament injuries account for up to 40 percent of all knee injuries, and of these, medial collateral ligament (MCL) injuries appear to be the most common $(24,4,21)$. I Bollen, et al. demonstrated that the incidence of combined ACL and MCL tear is approximately $30 \%$ of the incidence of ACL tears alone (29). A portion of these patients needs surgical reconstruction of the MCL. Also, some MCL injuries may not respond well to conservative treatment and require surgical treatment $(18,25)$.

There have been several techniques described for MCL reconstruction. However, these procedures are not without possible concurrent morbidity and issues, including extensive surgical exposure, donor site morbidity, loss of motion, non-anatomic graft placement, and technical complexity with doublebundle constructs $(22,23,4,1,18,10,29)$.

Marx et al introduced a new technique for MCL reconstruction that involved using an Achilles allograft. They reconstructed the MCL at the same time as ACL reconstruction (22). The authors reported advantages such as no donor site morbidity, secure fixation with bone-to-bone healing on the femur, small skin incisions, and isometric reconstruction (22).

Although Marx's technique has shown a satisfying outcome over time, some remaining issues necessitate the need for minor modifications.

In this technique, Achilles allograft is utilized without any bony attachment. Then the MCL is reconstructed in the first stage and other ligamentous injuries in the second stage when adequate knee ROM was achieved via rehabilitation. There are several benefits to this modified technique: 1) Knee ROM may improve by utilizing a two-stage technique with a physical therapy protocol initiated between stages. 2) The use of allograft tendon eliminates potential donor site morbidity, and fixing the graft with anchor sutures eliminates the fixation site irritation and avoids the need for hardware removal. 3) fixing the allograft in 2 places, the anatomical proximal and distal footprints of the superficial MCL, keeps the allograft in contact with the bone along the entire surface of the tibia. This may enhance tendon-bone healing and allows for more physiologic function due to achieving a more anatomic footprint.

Marx, et al. emphasized using tendon Achilles allograft with a calcaneal bone block to promote bone-bone healing. He used a screw and a washer for fixing the bony part of the allograft into the tibia (22). However, the medial proximal side of the leg has little subcutaneous coverage, and using screws and washer may cause hardware irritation necessitating future removal. To address this issue, anchor sutures were used to fix the allograft to the tibia. This technique minimizes the potential irritation of metallic devices under the skin.

Marx, et al. looked for the most isometric point for attaching the allograft on the tibia during surgery (22). Feeley, et al. performed a cadaveric study to determine the femoral and tibial fixation sites that would result in the most isometric MCL reconstruction technique (12). They concluded that MCL reconstruction performed with the femoral attachment of the MCL within the femoral footprint and the tibial attachment within the footprint of the MCL would result in the least graft excursion when the knee was cycled between $0^{\circ}$ and $90^{\circ}$ (12). In this technique, the anatomical footprint of the MCL was used for attachment rather than checking for the isometric site according to Feeley et al (12).

In another study Bin, et al. repaired or reconstructed medial or lateral ligament complexes in the first surgical stage within 2 weeks of injury. In the second stage, when the full range of motion was obtained 3 to 6 months later, they reconstructed the ACL and/or the PCL. They evaluated their outcomes based on stress radiographs, range of motion assessment, Lysholm score, Tegner activity stage, and International Knee Documentation Committee rating. All patients recovered full ROM, the mean Lysholm score was 87.6 points (range, 65 to 100 points), the mean Tegner stage was 3.9 (range 3 to 5 ) and the final overall IKDC rating were normal 
in 3 knees, nearly normal in 8, and abnormal in 4 . They concluded that the 2-stage surgical approach resulted in good outcomes in terms of the range of motion and stability (29). In these patients, MCL was reconstructed at the first and the ACL and/or the PCL remained intact to be repaired at the second stage. Patients did not experience any loss of ROM. Marx reported 2 cases of losses of $15^{\circ}$ of flexion. Although this difference may not be statistically significant, two-stage reconstruction may lead to a better range of motion.

The most important limitation of the current study was the low number of cases without a comparison group. Although the candidates for this type of reconstruction are few and other studies have reported similar numbers, a larger cohort is needed to conclusively demonstrate the benefits of this modification. Additionally, longer-term followup is required to assess the long-term efficacy of this technique.

In conclusion, patients with multi ligamentinjured knees, valgus laxity, and ROM were effectively restored through a 2-step surgical reconstruction. Patient-reported functional results were significantly improved postoperatively at the last follow-up. The modified Marx's techniques introduced in this article, would benefit patients by reducing metallic hardware irritation, and possibly restoring the full return of knee ROM and stability.

\section{List of abbreviations}

ACL : Anterior Cruciate Ligament

PCL : Posterior Cruciate Ligament

MCL : Medial Collateral Ligament

IKDC : International Knee Documentation Committee

ROM : Range of Motion

\section{REFERENCES}

1. Azar FM. Evaluation and treatment of chronic medial collateral ligament injuries of the knee. Sports medicine and arthroscopy review. $2006 ; 14(2)$ : 84-90.

2. Bin SI, Nam TS. Surgical outcome of 2-stage management of multiple knee ligament injuries after knee dislocation. Arthroscopy. 2007 ; 23(10) : 1066-72.

3. Bollen S. Epidemiology of knee injuries : diagnosis and triage. Br J Sports Med. 2000 ; 34(3) : 227-8.
4. Borden PS, Kantaras AT, Caborn DN. Medial collateral ligament reconstruction with allograft using a doublebundle technique. Arthroscopy. $2002 ; 18(4)$ : E19.

5. Bosworth DM. Transplantation of the semitendinosus for repair of laceration of medial collateral ligament of the knee. J Bone Joint Surg (Am) 1952 ; 34(1) : 196-202.

6. Brantigan OC, Voshell AF. The mechanics of the ligaments and menisci of the knee joint. J Bone Joint Surg (Am) 1941 ; 23(1) : 44-66.

7. Brantigan OC, Voshell AF. The tibial collateral ligament : its function, its bursae, and its relation to the medial meniscus. J Bone Joint Surg (Am) 1943 ; 25(1) : 121-31.

8. De Maeseneer M, Van Roy F, Lenchik L, Barbaix E, et al. Three layers of the medial capsular and supporting structures of the knee : MR imaging-anatomic correlation. Radiographics. 2000 ; 20 Spec No : S83-S89.

9. Edson CJ. Conservative and postoperative rehabilitation of isolated and combined injuries of the medial collateral ligament. Sports medicine and arthroscopy review. 2006 ; $14(2): 105-10$.

10. Fanelli GC, Tomaszewski DJ. Allograft use in the treatment of the multiple ligament injured knee. Sports medicine and arthroscopy review. 2007 ; 15(3) : 139-48.

11. Feeley BT, Muller MS, Allen AA, Granchi CC, et al. Biomechanical comparison of medial collateral ligament reconstructions using computer-assisted navigation. Am J Sports Med. 2009 ; 37(6) : 1123-1130.

12. Feeley BT, Muller MS, Allen AA, Granchi CC, et al. Isometry of medial collateral ligament reconstruction. Knee Surgery, Sports Traumatology, Arthroscopy. 2009 ; 17(9) : 1078-82.

13. Grood ES, Noyes FR, Butler DL, Suntay WJ. Ligamentous and capsular restraints preventing straight medial and lateral laxity in intact human cadaver knees. $J$ Bone Joint Surg (Am) 1981 ; 63(8) : 1257-69.

14. Indelicato PA. Non-operative treatment of complete tears of the medial collateral ligament of the knee. J Bone Joint Surg (Am) 1983 ; 65(3) : 323-9.

15. Kim SJ, Lee DH, Kim TE, Choi NH. Concomitant reconstruction of the medial collateral and posterior oblique ligaments for medial instability of the knee. J Bone Joint Surg (Br) 2008 ; 90(10) : 1323-1327.

16. Kovachevich R, Shah JP, Arens AM, Stuart MJ, et al. Operative management of the medial collateral ligament in the multi-ligament injured knee: an evidence-based systematic review. Knee Surg Sports Traumatol Arthrosc. 2009 ; 17(7) : 823-829.

17. La Prade RF, Engebretsen AH, Ly TV, Johansen S, et al. The anatomy of the medial part of the knee. J Bone Joint Surg (Am) 2007 ; 89(9) : 2000-10.

18. Larson RL. Combined instabilities of the knee. Clin Orthop Relat Res. 1980 ; (147) : 68-75

19. Lind M, Jakobsen BW, Lund B, Hansen MS, et al. Anatomical reconstruction of the medial collateral ligament and posteromedial corner of the knee in patients with 
chronic medial collateral ligament instability. Am J Sports Med. 2009 ; 37(6) : 1116-22.

20. Loredo R, Hodler J, Pedowitz R, Yeh LR, et al. Posteromedial corner of the knee : MR imaging with gross anatomic correlation. Skeletal Radiol. 1999 ; 28(6) : 305311.

21. Majewski M, Susanne H, Klaus S. Epidemiology of athletic knee injuries: A 10-year study. The knee. 2006 ; 13(3) : 184-8.

22. Marx RG, Hetsroni I. Surgical technique : medial collateral ligament reconstruction using Achilles allograft for combined knee ligament injury. Clin Orthop Relat Res. $2012 ; 1 ; 470(3): 798-805$.

23. Harding ML. W. Müller (Trans. T. G. Tegler) "The Knee : Form, Function and Ligament Reconstruction". Br J Sports Med. 1983 ; 17(3) : 214.

24. Phisitkul P, James SL, Wolf BR, Amendola A. MCL injuries of the knee : current concepts review. Iowa Orthop J. $2006 ; 26: 77-90$.

25. Robinson JR, Sanchez-Ballester J, Bull AM, Thomas Rde W, et al. The posteromedial corner revisited. An anatomical description of the passive restraining structures of the medial aspect of the human knee. J Bone Joint Surg (Br) $2004 ; 86(5): 674-81$.

26. Robinson JR, Sanchez-Ballester J, Bull AM, Thomas Rde W, et al. The posteromedial corner revisited. An anatomical description of the passive restraining structures of the medial aspect of the human knee. J Bone Joint Surg (Br) $2004 ; 86(5): 674-81$.

27. Torkaman A, Yazdi H, Hosseini MG. The Results of Single Bundle Versus Double Bundle ACL Reconstruction Surgery, a Retrospective Study and Review of Literature. Med Arch. 2016 ; 70(5) : 351-353.

28. Warren LF, Marshall JL. The supporting structures and layers on the medial side of the knee: an anatomical analysis. J Bone Joint Surg (Am) 1979 ; 61(1) : 56-62.

29. Wijdicks CA, Griffith CJ, Johansen S, Engebretsen L, et al. Injuries to the medial collateral ligament and associated medial structures of the knee. J Bone Joint Surg (Am) 2010 ; 92(5) : 1266-1280.

30. Yazdi H, Torkaman A, Ghahramani M, Moradi A, et al. Short term results of anterior cruciate ligament augmentation in professional and amateur athletes. $J$ Orthop Traumatol. 2017 ; 18(2) : 171-176. 